\title{
Latency and Packet Loss of an Interferred 802.15.4 Channel in an Industrial Environment
}

\author{
Jerker Delsing, Jens Eliasson and Viktor Leijon \\ EISLAB Luleå University of Technology \\ 97187 Luleå, Sweden \\ jerker.delsing@1tu.se, jens.eliasson@1tu.se, viktor.leijon@1tu.se
}

\begin{abstract}
There is currently a rapid development of new types of wireless communication channels for industrial automation. This paper aims to provide some experimental data and theoretical justification on packet latency and packet loss for a wireless communication channel exposed to intentional radio interference. The intentional radio interference used in the experiments is an attempt to simulate possible future co-existence scenarios in a dense wireless communication environment at an industrial site. For the cases tested packet loses of less than $10 \%$ was obtained. Latency is shown to depend on channel access and will has a deterministic behaviour.
\end{abstract}

Index Terms-Latency, packet loss, Interferred 802.15.4, Industrial environment

\section{INTRODUCTION}

The use of wireless devices in industrial environments is increasing. There still is a lot of doubt concerning the robustness and reliability of wireless communication in the industrial environment. This is particular if the communication is to be used for control where not only packet losses but also latency becomes of concern. Nevertheless, we see the emergence of wireless communication standards aimed at the industrial environment, the two most discussed standards are Wireless Hart [1] and ISA 100 [2]. We also find a number of wireless communication products directly tailored to industrial usage, one example is the Wisa technology from ABB [3], [4] other examples are available from Honeywell and others.

In nearly all these examples the radio frequency band is the free ISM band at $2.4 \mathrm{GHz}$. The major drawback of this is that numerous other applications have also chosen the same radio band.The three major commercial wireless radio technologies already established in that frequency band are WLAN (IEEE 802.11) [5], Bluetooth(IEEE 802.15.1) [6] and IEEE802.15.4 both which are part of the standardisation work under IEEE 802.15 [7], [8].

A lot of the previous research in this area addresses interference between WLAN and Bluetooth, and only the bandwidth capability of the wireless link has been examined. Interesting work on this has been made by Golmie [9] and others [10], [11], [12], [13]. Another important thing to know is to what extend we have radio activity in the ISM band, some very useful work has been performed by Biggs et.al. [14]. Most of this work targets office environments which at least so far are very different to industrial environments. In the 802.15.4 standard Annex E some basic considerations and data on coexistence is given [7].

Recent work also addresses coexistence of 802.15.4 and latency issues. Interesting work has been made by Bertocco and co-workers [15], [16], [17]. Here both some theoretical analysis of 802.15.4 latency paired with some experimental data is given. Despite these efforts we think there still is an issue on how a future massive radio usage in the ISM band will influence channel latency and packet losses for a 802.15 .4 radio.

The questions of interest for industrial automation use of wireless communication currently are:

- What radio "noise" do we presently find in a typical industrial environment?

- What interference will a massive future usage of WLAN, Bluetooth and 802.15.4 based radio communication cause to 802.15 .4 wireless communication?

- What characteristics will such interference give to data latency and packet losses?

In this paper, we present a background radio spectrum m;measurement from a real industrial environment. A lab environment has been setup to simulate a future scenario with massive radio communication in a limited physical space. 


\section{RADIO BACKGROUND EXPERIMENTAL SETUP}

To establish a baseline level of radio communications at an industrial site a series of background measurements have been made at different locations in an environment that is representative for the process industry. As a representative environment we have chosen Boliden Mineral AB's flotation plant in Garpenberg. Sweden. The background measurements were made using an Agilent spectrum analyzer $8560 \mathrm{~A}$ and E4440A, the antenna in all cases was Acconia HyperLOG 30180.

Three test locations were selected, two of them close to control loops handling level control in the flotation tanks of the zinc separation line. The measurement setup is shown in Figures 1 and 2. The third measurement was made close to the primary mill, which is equipped with a wireless serial communication link.

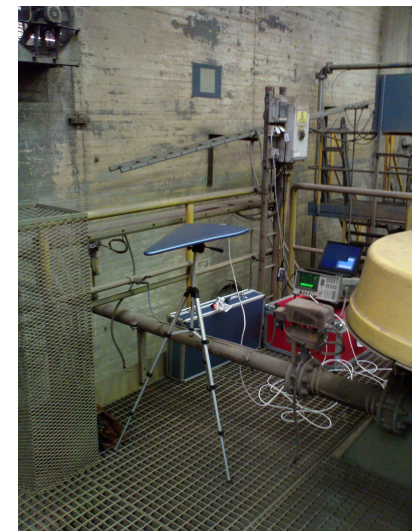

Fig. 1. Measurement setup at location 1 on top of a flotation tank in zinc separation line. To the left of the blue antenna we have the pressure sensor and to the right we have the control valve - these two together form the main control loop for the flotation tank.

At each measurement point eight different antenna locations were tested since the antena choosen is directive. Antenna directions were north, east, south and west, for each of these directions both horizontal and vertical polarizations was tested. All measurements showing very small differencies. Thus we judge the use of the log periodic antenna appropriate for the location.

The following measurements were made at the plant when in full operation:

1) Spectra $300-2.900 \mathrm{MHz}$, filter width $30 \mathrm{~Hz}$, for all 3 locations.

2) Spectra $2.400-2.500 \mathrm{MHz}$, filter width $10 \mathrm{kHz}$ for all 3 locations.

3) Spectra $2.400-2.500 \mathrm{MHz}$, filter width $10 \mathrm{kHz}, 2$ Bluetooth channels with intentional data/audio transmission, at locations 1 and 2 .

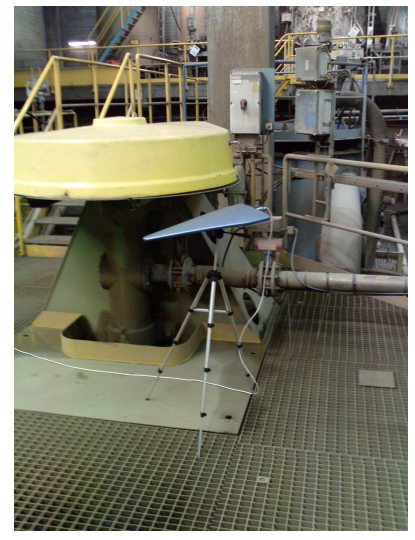

Fig. 2. Measurement setup at location 2 on top of a flotation tank in zinc separation line. Behind the blue antenna we have the control valve to the wright we have the pressure sensor for the control loop.

The noise floor for all these measurement was about $-100 \mathrm{dBm}$. In one of the data graphs, figure 5 the data plotting routine used indicated a seamingly higher noise floor. For the measurements with intentional Bluetooth communication locations 1 and 2 were used. Here Bluetooth communication was established between a standard laptop, IBM Thinkpad T60p, and two standard cell phones, Sony-Ericsson Z1010 and Sony-Ericsson P1i and one Bluetooth headset Sony Ericsson HBH610. Data transfer over Bluetooth was made using file transfer and audio streaming. File transfer was made from the P1i to the laptop using $20 \mathrm{MB}$ audio files. Audio transfer was generated between the Z1010 and the HBH610 by playing voice mail messages with a duration of over four minutes.

\section{LAB EXPERIMENTAL SETUP}

A lab setup aimed at simulating a future radio environment has been developed. The lab setup is located to our anechoic chamber which is radio "silent" up to $18 \mathrm{GHz}$ [18]. The aim of this setup is to enable tests of wireless communication in the $2.4 \mathrm{GHz}$ ISM band with co-existing radio communication. Currently the lab setup does not aim for handling effects like reflections, multipath, Doppler spread etc.

\section{A. Test channel setup}

Two Mulle wireless sensor network devices [19], [20] using Atmel IEEE 802.15.4 transceivers were used in order to measure changes in packet loss and latency with changing radio conditions. In this configuration the Mulle device is capable of running the 802.15.4 radio protocol with or without 
retransmissions. TinyOS were used as an operating system for the Mulles. TinyOS includes a driver for the Atmel AT86RF230 transceiver found on the Mulle v5.2 platform.

\section{B. Latency and packet loss analysis}

Some basic analysis can be made regarding the causes for packet loss and latency over the 802.15.4 communications channel. The latency of the 802.15.4 channel can be divided into a series of contributions causes. The following steps contribute to the over all latency:

- MAC handing:

This is time spent in the node, doing the processing needed for the communication. This time is considered small compared to all other delays.

- Transmission buffers:

The M16c62p processor on the Mulle fills the buffer of the 802.15.4 transceiver rf230 through a soft SPI bus and then enables the transmission from the 802.15.4 transceiver. The measured byte transfer time here is 65 $\mu$ s per byte

- Radio transmission:

The basic transfer time can be calculated from the bandwidth of the 802.15.4 transceiver, 250kbit/s. $32 \mu$ s per byte.

- Receiving buffers:

The M16c62p processor of the Mulle receives the notification from the 802.15.4 transceiver and reads data from its buffer through the soft SPI bus. We here have the same data as for sending i.e. $65 \mu$ s/byte

- Mac handing:

This time is again mainly spent on processing network protocol and is again assumed to be small compared to other latency.

For a given packet size we thus get the following basic transfer latency:

$$
t_{\text {latency }}=p_{\text {size }} *(2 * 65)+32 *\left(p_{\text {size }}+12\right) \mu s
$$

where $p_{\text {size }}$ is the packet size including the 802.15.4 address but excluding 6 byte PHY and 6 byte MAC overhead as given section 6.3 and 7.2 of the 802.15.4 standard [7]. As an example this gives us $4.1 \mathrm{~ms}$ latency for a payload of 23 byte. This is the minimal latency for sending data from an application in the sensor network node until its received by another application at the receiving network node.
We can now take into consideration that there may be interference on the radio channel. The 802.15.4 standard prescribes certain rules for how the medium can be accessed, since the protocol uses CSMA/CA this mainly consists of a series of clear channel assessments interlaced with backoffs. For consistency with the standard we express the latency in number of symbol times. One bit is $4 \times$ symboltime in the $250 \mathrm{kbit}$ variant, to get seconds instead the result can be divided by 62500 . A help variable $m$ is used to detect if we reach the maximum number of allowed retries in accessing the channel:

$$
\begin{gathered}
m=\min (\operatorname{macMaxBE}-\operatorname{macMinBE}, \\
\text { macMaxCSMABackoffs })
\end{gathered}
$$

where macMaxBE is 5 (the allowed range is 3-8), macMinBE is $3(0-3)$, and macMaxCSMABackoffs is $4(0-5)$. The maximum total latency maxTotalWait in symbol times then becomes :

maxTotalWait $=$ aUnitBackoffPeriod $\times$

$$
\begin{aligned}
& \left(\sum_{k=0}^{m} 2^{k}+\left(2^{\operatorname{macMaxBE}}-1\right)\right. \\
& \quad \times(\text { macMaxCSMABackoffs }-m)) \\
& +8 \times \text { macMaxCSMABackoffs }
\end{aligned}
$$

where a UnitBackoffPeriod is 20 symbol periods.

To this comes the maximum transfer time maxSendTime (also expressed in number of symbol times).

$$
\max \text { SendTime }=24+2 \times \text { packetSize }
$$

where packetSize is the number of bytes transferred. In addition to this we have the maximum transfer time, maxSendTime, which is two times the number of bytes when expressed in symbol times (since each byte is mapped onto two symbols).

\begin{tabular}{|l|r|}
\hline Probability of back-off & no of back-offs \\
\hline $80 \%$ & 0 \\
\hline $10 \%$ & 1 \\
\hline $5 \%$ & 2 \\
\hline $3 \%$ & 3 \\
\hline $2 \%$ & 4 \\
\hline
\end{tabular}

TABLE I

THE CCA FAILURE PROBABILITY USED FOR OUR SIMULATIONS OF THE CSMA-CA ROUTINE IN 802.15.4.

We have created a small script to simulate the effects of back offs on the transmission times. If we 
assume that the number of back-offs for each 100 byte packet is given by the distribution in Table I we get the latency distribution shown in Figure 3.

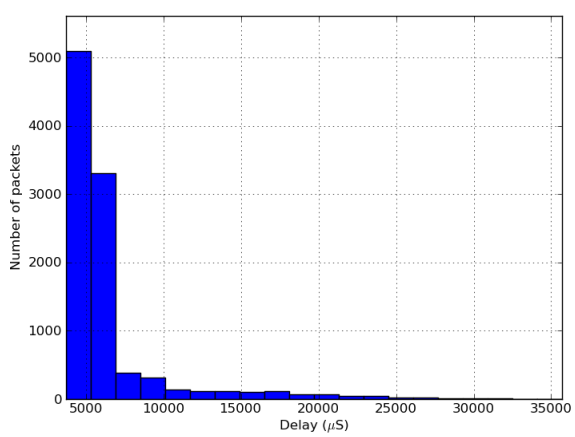

Fig. 3. Simulated latency distribution for 802.15.4, given the back off distribution given in Table I.

\section{Test channel measurements}

To simulate a future radio communication scenario based on current control period data control paradigm we did design the following tests. For each test, the sender node transmitted a fixed number of packets to the receiver with a variable interval. A time interval of $10 \mathrm{~ms}$ reflecting medium fast sampling time in industrial control applications were selected. Each packet contained a sequence number, a time stamp, and a payload. The sequence number was used to detect packet loss, and the time stamp was used to measure the total time required to transmit a packet. The total time includes sending the packet over a SPI port to the transceiver, then over the air to the receiving module, which will send it again over a serial port to the receiving program. Thus the data latency is defined as time from application sending data till it is received by an application at the receiving terminal. The processing time at the sending and the receiving Mulle modules are thus included. This total processing time is currently unknown since its dependent on software in the Atmel 802.15.4 transceiver. This causes an uncertainty when trying to analyse the latency data obtained. Data obtained though indicates that this uncertainty does not mask the basic behaviour of the communication channel.

Three packet sizes have been used for the test channel, with different payload sizes and a standard TinyOS ActiveMessage header are:

- Small packets - 27 bytes.

- Medium packets - 77 bytes.

- Large packets - 127 bytes.
In order to measure the latency with a very fine granularity, the transmitter and receiver Mulles were using a master clock of $1 \mathrm{MHz}$ generated by a third Mulle. The clock gives a a maximum error of 1 us since the Mulle which provides master both generates the clock and starts the the transmitter and receiver Mulles simultaneously. This approach guarantees that the transmitter and receiver nodes are perfectly synchronized within one tick of the master clock. To measure the total time necessary for transmitting a packet between the application layer of the transmitter and receiver nodes, the transmitter reads the master clock, which is captured in a free running 16-bit counter, and adds the current time stamp to the packet's payload. It also adds a packet number which is used to detect packet loss. Whenever a packet is received at the receiver, it also reads the master clock and calculates the total packet latency. The latency is then printed together with the packet id on a debug USB serial port, which in turn is connected to a PC. The PC stores all debug messaged and can generate reports regarding packet loss and latency statistics.

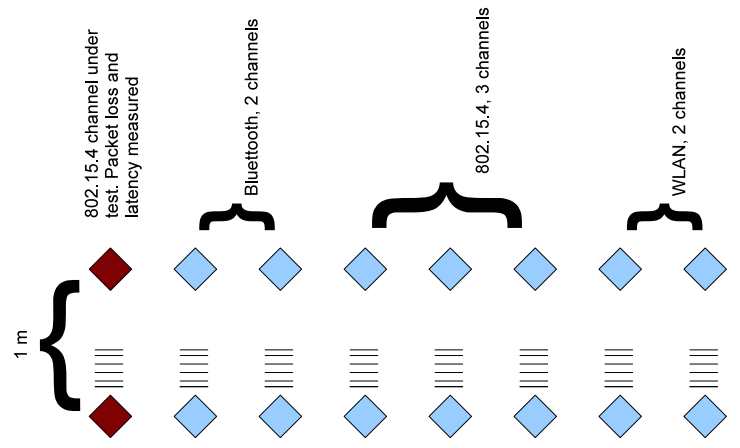

Fig. 4. Physical layout of the test environment for the simulated future radio environment.

\section{Simulated radio and IEMI environment}

To simulate what a future radio environment may look like we have equipped that lab with seven background nodes, sending over the wireless band. Of these we have two WLAN nodes, two Bluetooth 1.1 nodes and three 802.15.4/Zigbee nodes. For the WLAN and 802.15.4 channels the channel frequency can be changed. Bluetooth 1.1 was chosen since the 1.1 version does not have the interference avoidance function which is present in the Bluetooth 1.2 specifications. The physical layout of the simulated radio environment is shown in figure 4.

Using different frequency settings we can generate a large variety of interferences to the wireless 
channel that we want to test. These interferences office or industrial environment with normal use of wireless communication.

Each of the interference channels can be fed with any type of communication. For the experiments in this paper we chose to send a steady stream of data over each channel. For the Bluetooth channel we used a steady ping stream with an interval of $10 \mathrm{~ms}$ between each packet, and for the 802.15.4 channel we sent a 100 byte package every five milliseconds.

For the WIFI we chose a flooding ping, where we did not wait for response before sending the next one, having up to one hundred outstanding Test 9 packets packets, using a 1000 byte packet size.

The following latency and packet loss measurements have been performed using the Mulle, see Section III-A, for the test channel. The whole test setup was placed in our anechoic chamber, thus we know that no external radio interference will influence the measurements. For the tests a varietyT of of out-of-band and in-band interference were generated.

We had the following test cases:

Test 1 No interference: packets size 27 bytes This was to get to get a base measurement.

Test 2 Low level of interference: packets size 27 bytes Test channel at $2.405 \mathrm{GHz}$, two WLAN channels at 2.447 and $2472 \mathrm{GHz}$ and three ZigBee transmitters at $2.440 \mathrm{GHz}$ all clearly out of band to the test channel in addition to the two Bluetooth transmitters. are of a type that we are likely to encounter in an

close-band high bandwidth interferences.

Test 6 Check scenario I: packets size 27 bytes

Test channel at $2.405 \mathrm{GHz}$, WLAN at 2.417 $\mathrm{GHz}$ and $2.472 \mathrm{GHz}$, three ZigBee transmitters at $2.440 \mathrm{GHz}$ and two Bluetooth transmitters. Here we have one close band high bandwidth interference channel.

Test 7 Check scenario II: packets size 27 bytes Test channel at $2.405 \mathrm{GHz}$, three ZigBee transmitters at $2.405 \mathrm{GHz}$. Here we have and in-band low bandwidth interference channels.

Test 8 No interferences: packet size 77 bytes Another base band measurement Low level of interference: packets size 77 bytes

Test channel at $2.405 \mathrm{GHz}$, two WLAN channels at 2.447 and $2472 \mathrm{GHz}$ and three ZigBee transmitters at $2.440 \mathrm{GHz}$ all clearly out of band to the test channel, in addition to the two Bluetooth transmitters.

Worst case scenario: packets size 77 bytes Test channel at $2.405 \mathrm{GHz}$, two WLAN channels at 2.417 and $2472 \mathrm{GHz}$, three ZigBee transmitters at $2.405 \mathrm{GHz}$ and two Bluetooth transmitters. Here we have one in-band low bandwidth interference channel and two close band high bandwidth interferences.

Test 11 Check scenario I: packets size 77 bytes Test channel at $2.405 \mathrm{GHz}$, WLAN at 2.417 $\mathrm{GHz}$ and $2.472 \mathrm{GHz}$, three ZigBee transmitters at $2.440 \mathrm{GHz}$ and two Bluetooth transmitters. Here we have one close band high bandwidth interference channel.

Test 3 Medium level of interference: packets size 27Test 12 Worst case scenario: packets size 127 bytes bytes

Test channel at $2.405 \mathrm{GHz}$, two WLAN channels at 2.412 and $2417 \mathrm{GHz}$. Here we have two close in-band interference high bandwidth channels.

Test 4 Medium level of interference: packets size 27 bytes

Test channel at $2.405 \mathrm{GHz}$, two WLAN channels at 2.412 and $2417 \mathrm{GHz}$. In addition we have three ZigBee transmitters at $2.440 \mathrm{GHz}$ and 2 Bluetooth transmitters. Here we have a out of band low bandwidth channel and two close in-band interference high bandwidth channel.

Test 5 Worst case scenario: packets size 27 bytes Test channel at $2.405 \mathrm{GHz}$, two WLAN channels at 2.417 and $2472 \mathrm{GHz}$, three ZigBee transmitters at $2.405 \mathrm{GHz}$ and two Bluetooth transmitters. Here we have a in-band low bandwidth interference channel and two
Test channel at $2.405 \mathrm{GHz}$, two WLAN channels at 2.417 and $2472 \mathrm{GHz}$, three ZigBee transmitters at $2.405 \mathrm{GHz}$ and two Bluetooth transmitters. Here we have two in-band low bandwidth interference channel and two close band high bandwidth interferences.

\section{RESUlTS}

The general background radio environment found at the Boliden Garpenberg flotation plant is given in figure 5. No major differences were found for horizontal or vertical polarization or for different locations at the three test places. In Figure 6 we find a representative spectrum for the ISM band at the site.

For the lab tests we obtained latency and packet loss data as follows: The minimum latency for three different packet sizes indicates that the minimum latency is linear to the packet size and about $95 \mu \mathrm{s} /$ byte. This is illustrated in figure 7 . 


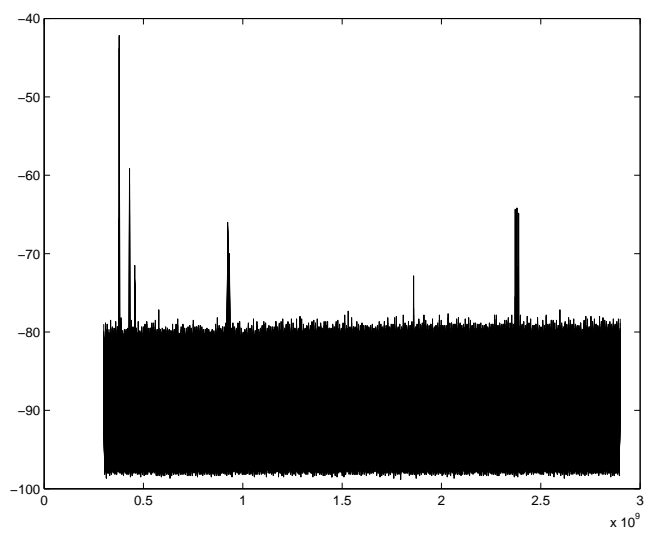

Fig. 5. General radio background, $300-2.900 \mathrm{MHz}$, horizontal polarization. GSM, 3G and some minor WLAN traffic is found. Below $500 \mathrm{MHz}$ serial data modem traffic and emergency/military traffic is found.

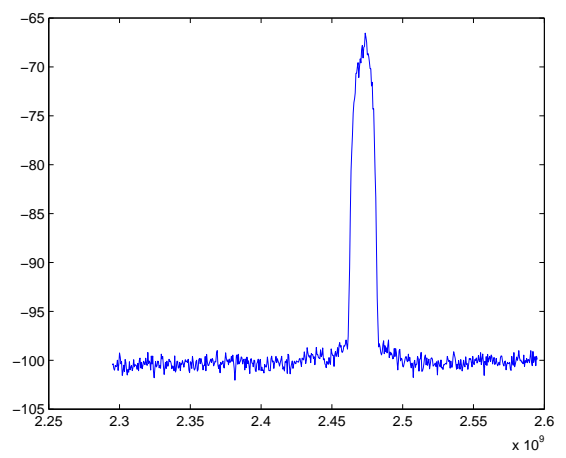

Fig. 6. General radio background in the ISM band, horizontal polarization. In this case we do see one of the interferrence channels in the ISM band. With no intentional interferrence we found no additional ISM devices transmitting and a noise flow of at least $-100 \mathrm{dBm}$

The packet loss under undisturbed conditions is zero, as expected. For close band interference from adjacent WLAN channels we get a small packet loss. When adding in-band 802.15.4 channel interference the packet loss is increased. Losses also increase with packet sizes, see figure 8 . Since only 3 packet sizes a more thorough investigation of the reason for the apparent bend at 90 byte packet is not feasible.

The maximum latency is clearly increasing with severity of interference. This can be seen from figure 9.

Another way of viewing the data is the maximum latency increase for the different tests. In Figure 10 the max latency increase is shown relative to base tests 1 and 2. For the average latency over 10.000 packets we find small $(<1 \%$ [27 byte] and $<2.4 \%$ [127 byte packets]) influences due to

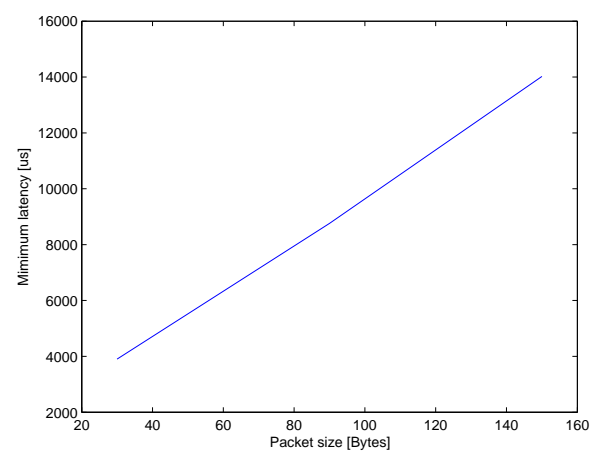

Fig. 7. Packet latency in $\mu$ s as a function of packet size.

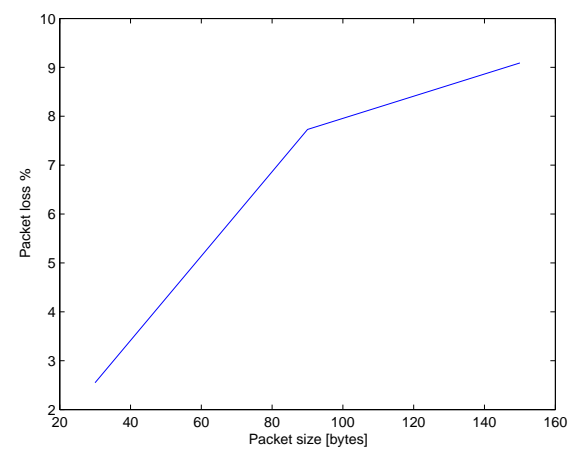

Fig. 8. Packet losses in percent as a function of packet size.

interferences.

\section{DISCUSSION}

From the background measurements we can conclude that the radio environment at the $2.4 \mathrm{GHz}$ band in an industrial environment today is relatively unused. Occasional WLAN and Bluetooth communication show up but will be limited both in area and duration. It was clear that the Bluetooth communication came from personnel having cell phones and Bluetooth headsets. No ZigBee communication was detected and only rarely was WLAN communication detected. To our knowledge only WLAN is installed at the plant but with a very low utilization.

For a future radio environment as tested in the lab we can conclude that bare 802.15.4 communication does not show any severe packet losses under quite heavy interferences from both in-band and close band sources. Out of band interference in the $2.4 \mathrm{GHz}$ band does not give any packet loss or increased latency. We find some increases in latency for in-band 802.15.4 radio traffic. For close band heavy WLAN traffic we find an even 


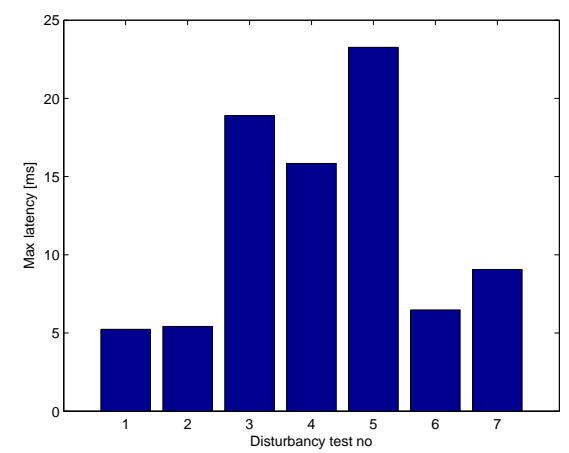

Fig. 9. Maximum latency shown for each test case, for a fixed packet size.

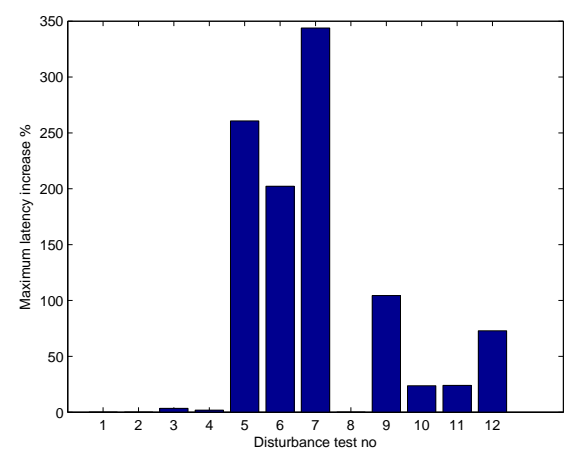

Fig. 10. Maximum latency increase shown for each interference test normalised to the base tests 1 and 2 for packet sizes 27 and 77 bytes. Since packet size 127 in test 8 did not have a base test, test 8 is shown as zero.

greater increase in latency. In the worst case a sixfold increase in latency was recorded. The reason for the latency increases is most likely found in the CSMA-CA PHY/MAC layer scheme of the 802.15.4 radio (the packet retransmission mechanism was disabled for all tests). On a congested channel the the CSMA-CA will delay transmission based on a random exponential back-off algorithm. The latency induced by this is dependent on control parameters of the 802.15.4 transceiver.

The issue of packet loss is dependent on both excess latency and errors in the received data. The cut-off for when latency becomes packet loss depends on the maximal number of back-offs in the system. The amount of errors in the received data is determined by the receiver using the 802.15.4 CRC mechanism [7]. For data with transmission errors there is a retransmit mechanism in 802.15.4 [7]. The drawback is that such retransmissions will further increase latency.

The implication of this data to other PHY/MAC layer schemes is as follows. The super frame and guaranteed time slot (GTS) mechanism in 802.15.4 [7] as used in WirelessHART [1] and ISA 100 [2] will have a packet loss when a packet is not transmitted within the time slot given to each process. The reason for a non-transfer under interferred conditions will then only be based on reception of packets with errors in them, for which 802.15.4 provides only a CRC check mechanism in the MAC layer. This implies that if the packet in a time slot is interferred the channel latency is determined by the handling of the 802.15.4 super frame scheme.

\section{CONCLUSIONS AND FURTHER WORK}

Based on the experiments made it is clear that extreme radio interference will cause packet loss for the 802.15.4 channel that was tested. The latency distribution is also clearly affected by the radio interferences. This has implications for both the CSMA/CA and Superframe/GTS media access mode of 802.15.4 regarding both latency and packet losses.

For the three question we put forward in this paper we can conclude the following:

- Currently the radio noise in the $2.4 \mathrm{GHz}$ ISM band is very limited.

- A future massive usage of wireless technology in the $2.4 \mathrm{GHz}$ ISM will cause moderate package losses and thus limit the available bandwidth. Further, channel latency will also be moderately affected.

- Packet losses is most likely randomly distributed in time within the time frame of the interference. The same holds for channel latency.

\section{REFERENCES}

[1] (2008, April) Wireless hart. Web. [Online]. Available: http://www.hartcomm2.org/

[2] (2008, April) Isa 100. ISA Web. [Online]. Available: http://www.isa.org/

[3] R. Steigmann and J. Endresen, "White paper, introduction to wisa v2.0," ABB, Tech. Rep., 2006.

[4] D. Dzung, J. Endresen, J.-E. Frey, and C. Apneseth, "Design and implementation of a real-time wireless sensor/actuator communication system," in Proc. IEEE Symposium on Emerging Technologies and Factory Automation, ETFA 2005, vol. 2, 2005, pp. 433-442.

[5] "Wireless local area network," Wikipedia, 2008. [Online]. Available: http://en.wikipedia.org/wiki/IEEE_802.11

[6] (2008, April) Bluetooth. Wikipedia. [Online]. Available: http://en.wikipedia.org/wiki/Bluetooth

[7] $(2009, \quad$ Dec) Ieee 802.15 wpan task group 4 (tg4). Wikipedia. [Online]. Available: http://www.ieee802.org/15/pub/TG4.html

[8] $(2009$, Dec) Ieee 802.15 working group for wpan. Wikipedia. [Online]. Available: http://ieee802.org/15/index.html 
[9] N. Golmie, "Bluetooth dynamic scheduling and interference mitigation," Mobile Networks and Applications, vol. 9, pp. 21-31, 2005.

[10] J.-A. Park, S.-K. Park, D.-H. Kim, P.-D. Cho, and K.-R Cho, "Experiments on radio interference between wireless lan and other radio devices on a $2.4 \mathrm{ghz}$ ism band," Vehicular Technology Conference, 2003. VTC 2003-Spring. The 57th IEEE Semiannual, vol. 3, pp. 1798-1801 vol.3, 22-25 April 2003.

[11] T. Keller and J. Modelski, "Experimental results of testing interferences in $2.4 \mathrm{ghz}$ ism band," in Proc. 33rd European Microwave Conference, 2003, pp. 1043-1046.

[12] W. Feng, A. Nallanathan, and H. Garg, "Impact of interference on performance of bluetooth piconet in $2.4 \mathrm{ghz}$ ism band," Electronics letters, vol. 38, no. 25, Dec 2002.

[13] M. Chandrashekhar, P. Choi, K. Maver, R. Sieber, and K. Pahlavan, "Evaluation of interference between ieee $802.11 \mathrm{~b}$ and bluetooth in a typical office environment," Personal, Indoor and Mobile Radio Communications, 2001 12th IEEE International Symposium on, vol. 1, pp. D-71-D-75 vol.1, Sep 2001.

[14] M. Biggs, A. Henley, and T. Clarkson, "Occupancy analysis of the $2.4 \mathrm{ghz}$ ism band," in IEE ProceedingsCommunications, vol. 5, no. 151. IEE, 2004, pp. 481488.

[15] M. Bertocco, G. Gamba, A. Sona, and S. Vitturi, "Experimental characterization of wireless sensor networks for industrial applications," IEEE Transaction on Instrumentation and Measurement, vol. 57, no. 8, pp. 1537-1546, August 2008.

[16] M. Bertocco, G. Gamba, and A. Sona, "Is csma/ca really efficient against interference in a wireless control system? an experimental answer," in Proc. Emerging Technologies and Factory Automation, 2008. ETFA 2008. IEEE International Conference on, sept. 2008, pp. 885 -892.

[17] C. D. Dominicis, P. Ferrari, A. Flammini, E. Sisinni, M. Bertocco, and G. G. andC. Narduzzi andF. Tramarin, "Investigating wirelesshart coexistence issues through a specifically designed simulator," in Proc IEEE IMTC, May 2009

[18] S. Inc., "Delivery test report for eislab emc chamber," Sippell Inc., Tech. Rep., 1997.

[19] J. Johnasson, M. Völker, J. Eliasson, Å. Östmark, P. Lindgren, and J. Delsing, "Mulle:a minimal sensor networking device - implementation and manufacturing challenges," in Proc. IMAPS Nordic 2004. IMAPS, Sept. 2004.

[20] (2010, April) Eistec. [Online]. Available: www.eistec.se 